$\mathbb{T}$ periodica polytechnica

Architecture

40/1 (2009) 3 - 8

doi: 10.3311/pp.ar.2009-1.01

web: http://www.pp.bme.hu/ar

(c) Periodica Polytechnica 2009

RESEARCH ARTICLE

\section{Countinuous and discrete models for} abrasion processes

\author{
Gábor Domokos / András Á Sipos / Péter L. Várkonyi
}

Received 2009-03-19

\begin{abstract}
The origin of the shapes of stones and other particles formed by water or wind has always attracted the attention of geologists and mathematicians. A classical model of abrasion due to W. J. Firey leads to a geometric partial differential equation representing the continuum limit of the process. This model predicts convergence to spheres from an arbitrary initial form; analogously, the two-dimensional version of the model predicts convergence to circles. The shapes of real stones are, however, not always round. Most notably, coastal pebbles tend to be smooth but somewhat flat, and ventifacts (e.g. pyramidal dreikanters) often have completely different shapes with sharp edges. Inspired by Firey's results, a new PDE is derived in this paper, which not only appears to be a natural mathematical generalization of Firey's PDE, but also represents the continuum limit of a genezalized abrasion model based on recurrent loss of material due to collisions of nearby pebbles. We also introduce a related, mezo-scale discrete random model which is ideally suited for analyzing wear processes in specific geometric scenarios. Preliminary results suggest that our model is capable to predict a broad range of limit shapes: polygonal shapes with sharp edges develop due to sand blasting (big stone surrounded by infinitesimally small particles), round stones emerge due to collisions with relatively big stones, and flat shapes are the typical outcome in the intermediate case. The results show nice agreement with real data despite the model's simplicity.
\end{abstract}

\section{Keywords}

surface evolution $\cdot$ curvature $\cdot$ ventifact

\section{Acknowledgement}

The authors thank Wesley Pegden for useful discussions. This work has been supported by OTKA grant 72146.

Gábor Domokos

András Á Sipos

Péter L. Várkonyi

Department of Mechanics, Materials and Structures, BME, H-1111 Budapest Múegyetem rkp. 3., Hungary

\section{Introduction}

The abrasion of an object is the result of many small, however discrete mechanical impacts. Its models can be classified according to the amount of detail they include; one can distinguish between three categories:

1 Macro-scale averaged continuum models, based on partial differential equations (PDEs)

2 Mezo-scale models, based on averaged discrete events (each of which represents the effects of many impacts)

3 Micro-scale models, based on discrete, individual impacts

While each category is important in its own right, the choice depends on the goals. As we move from macro-scale towards micro-scale, we trade qualitative insight for quantitative accuracy. Macro-scale models are most suitable to achieve global, qualitative results on general abrasion processes, mezo-scale models, while offering fair quantitative agreement, are optimal to determine the qualitative behaviour in specific situations and environments and micro-scale models are best equipped to obtain quantitative results, however, often fail to provide wellfounded qualitative insight.

Since we are primarily interested in the latter we will first describe a general macro-scale model, extending previous models to a more general abrasion process in the form of a PDE, then we proceed to present a closely related mezo-scale model. While the exact mathematical relationship of these two models needs still to be clarified, they undoubtedly correspond to closely related physical processes, which accounts for their similar (though as we show later, not identical) behaviour. Our goal is merely to describe these models, applications are beyond the scope of the present paper.

One of the best-known PDE model of the abrasion process of pebbles has been proposed by Firey (1974) [1]. He considered pebbles losing small portions of their volumes via successive collisions with a plane representing the underlying ground. If the orientation of the colliding pebbles is assumed to be random with uniform distribution, the continuous limit of this process is surface wear with speed proportional to curvature (in 3D: Gaussian curvature; in 2D: scalar curvature, see Section 2) provided 
that the initial shape is convex. Not surprisingly, this process quickly abrades sharp vertices with high curvature, and arbitrary initial shapes become spheres in 3D (Andrews, 1999) or circles in 2D (Gage, 1984, Gage \& Hamilton, 1986) [2-4] as they gradually contract to points. Our models consider collisions to finite-sized impactors, and Firey's model emerges as a special case: when the abraders are much bigger than the object in question.

While real pebbles typically do get smooth, they are usually not perfect spheres. Some deviation follows from the discreteness of abrasion (Durian et al, 2006, [5] see also Section 4.) nevertheless the fact that coastal pebbles tend to be flat (Rayleigh, 1944, Wald, 1990, Lorang \& Komar, 1990) [6-8] calls for a different explanation. Dobkins \& Folk (1970) [9] found that pebbles on Tahiti are sometimes assorted by the wave current and flat ones accumulate on the beach. In some other cases, the flatness reflects the stratified microstructure of the material. According to an alternative explanation, initially flat pebbles collide with the waterbed with non-uniformly distributed orientations which probably helps maintaining/increasing their flatness. It was also proposed by Dobkins \& Folk (1970) [9] that the presence of sand plays important role in flattening pebbles. Probably all these factors contribute to the formation of observed pebble shapes. The inclusion of some factors is beyond the scope of a simple partial differential equation (PDE), nevertheless the effect of 'sand blasting' is easy to approximate in the spirit of Firey's PDE model. In Section 3 we generalize Firey's 2D model by considering collisions with nearby pebbles instead of planes. Our general PDE model includes both Firey's model and also abrasion by infinitesimally small sand particles as special cases. The continuous limit of the new model in $2 \mathrm{D}$ is derived: abrasion speed is $p \rho+1$ where $\rho$ is curvature, and $p$ is a constant. In particular, sand blasting corresponds to $p=0$, Firey's model to $\mathrm{p} \rightarrow \infty$. An analogous expression for the 3D model is also discussed briefly without computational details. In Section 4, we introduce a discrete version of this model. We describe two events (type (A) and (B)), which are analogous to the two extremal cases of the continuum model ( $p=0$ and $p \rightarrow \infty$ ). While not a straightforward discretizations of the PDE, the discrete mezo-scale model in Section 4 provides similar predictions though not for all scenarios. Some predictions of the models and their physical relevances are discussed in Section 5.

\section{Firey's PDE model}

The 2D version of the abrasion model of Firey (1974) assumes that a convex stone represented by a closed, convex plane curve $S$ repeatedly collides with a straight line $S^{*}$. During each collision, a small fracture of the stone gets lost at the point of contact. We consider the process in a coordinate system fixed to $\mathrm{S}$, see reference axis $x$ and reference point $\mathrm{O}$ in Fig. 1 (left). Each collision is parameterized by $\beta$ denoting the angle of the normal vector of $\mathrm{S}^{*}$ relative to $x$. We assume that $\beta$ is a uniform random variable on $[0,2 \pi]$, i.e collisions with orientation
$\beta_{0}<\beta<\beta_{0}+\Delta \beta$ happen with mean frequency $f \Delta \beta$ ( $f$ is a constant). The average area $\delta$ lost by the pebble $\mathrm{S}$ is assumed to be independent of the location of the collision.

We consider an infinitesimal piece of length $\Delta s$ on which the angle $\phi$ of the inward pointing normal unit vector $\mathbf{n}_{\phi}$ of $\mathrm{S}$ to axis $x$ is $\phi \in(\alpha-\Delta \alpha / 2, \alpha+\Delta \alpha / 2)$. In a collision, $\Delta s$ is hit by the plane $\mathrm{S}^{*}$ if $\alpha-\Delta \alpha / 2 \leq \beta \leq \alpha+\Delta \alpha / 2$ (modulo $2 \pi$ ). The mean frequency of such collisions is $f \Delta \alpha$, hence the mean area loss per unit time is $\delta f \Delta \alpha$. The mean speed of wear is then $\delta f \Delta \alpha / \Delta s=\delta f \rho(\alpha)$ where $\rho(\alpha)$ is the curvature of the curve. Thus, in the continuum limit, the abrasion process leads to the geometric PDE

$$
\dot{\mathbf{v}}=\delta f \rho(\alpha) \cdot \mathbf{n}_{\alpha}
$$

where $\mathbf{v}$ is the position vector of a point on the perimeter with (time-dependent) normal direction $\alpha$; dot denotes derivative with respect to time $t$. Hence, abrasion speed is proportional to curvature. The only possible limit shape of the curve (after scaling) is a circle, as already pointed out in Section 1. The same arguments in 3D lead to surface contraction proportional to Gaussian curvature and convergence to spheres.

\section{The generalized PDE model}

Here we derive a natural, physical generalization of Firey's model, where the straight line $\mathrm{S}^{*}$ is replaced by another convex curve (Fig. 1 right). Let $x, x^{*}, \mathrm{O}, \mathrm{O}^{*}$ denote reference axes and reference points of the two respective curves. We keep the notations of Section 2; in addition, $\rho^{*}(\phi)$ denotes the curvature of $\mathrm{S}^{*} . \mathrm{S}^{*}$ is assumed to be subject to straight motion relative to $\mathrm{S}$; a collision of the two curves is parameterized by 3 random parameters: $\beta$ determines the angle between $\mathrm{x}$ and $x^{*}$; angle $\gamma$ denotes the direction of motion of $\mathrm{S}^{*}$ relative to $x$; finally $d$ denotes the distance of the straight orbit of $\mathrm{O}^{*}$ from $\mathrm{O} . \Delta s^{*}$ is the interval on $\mathrm{S}^{*}$ where the angle of the outward-pointing normal of the curve is $\phi \in(\beta+\alpha-\Delta \alpha / 2, \beta+\alpha+\Delta \alpha / 2)$ to $x^{*}$ (Fig. 1, right).

As in Section 2, it is assumed that $d, \beta, \gamma$ are uniformly distributed: collisions with $\gamma, \beta, d$ lying in given intervals of sizes $\Delta \beta, \Delta \gamma$ and $\Delta d$, respectively occur with mean frequency $f \Delta \beta \Delta \gamma \Delta d$. The pebble $\mathrm{S}$ gets hit at $\Delta s$ if the parameters fall in appropriate ranges: $S^{*}$ needs to be between the two extreme positions shown in Fig. 1 (right) at the instant of the collision. One of these copies of $\mathrm{S}^{*}$ can be transformed to the other by shifting it by distance

$$
\Delta s+\Delta s^{*}=\left(\frac{1}{\rho(\alpha)}+\frac{1}{\rho^{*}(\alpha-\beta)}\right) d \alpha
$$

in direction perpendicular to $\mathbf{n}_{\alpha}$. For fixed $\beta$ and $\gamma$, this displacement corresponds to an interval in parameter $d$ of size

$$
\begin{gathered}
\Delta d(\beta, \gamma)=\left(\Delta s+\Delta s^{*}\right) \cos (\alpha-\gamma)= \\
\left(\frac{1}{\rho(\alpha)}+\frac{1}{\rho^{*}(\alpha-\beta)}\right) d \alpha \cos (\alpha-\gamma)
\end{gathered}
$$

if $\cos (\alpha-\gamma)>0$, and there is no such interval if $\cos (\alpha-\gamma) \leqslant 0$. 


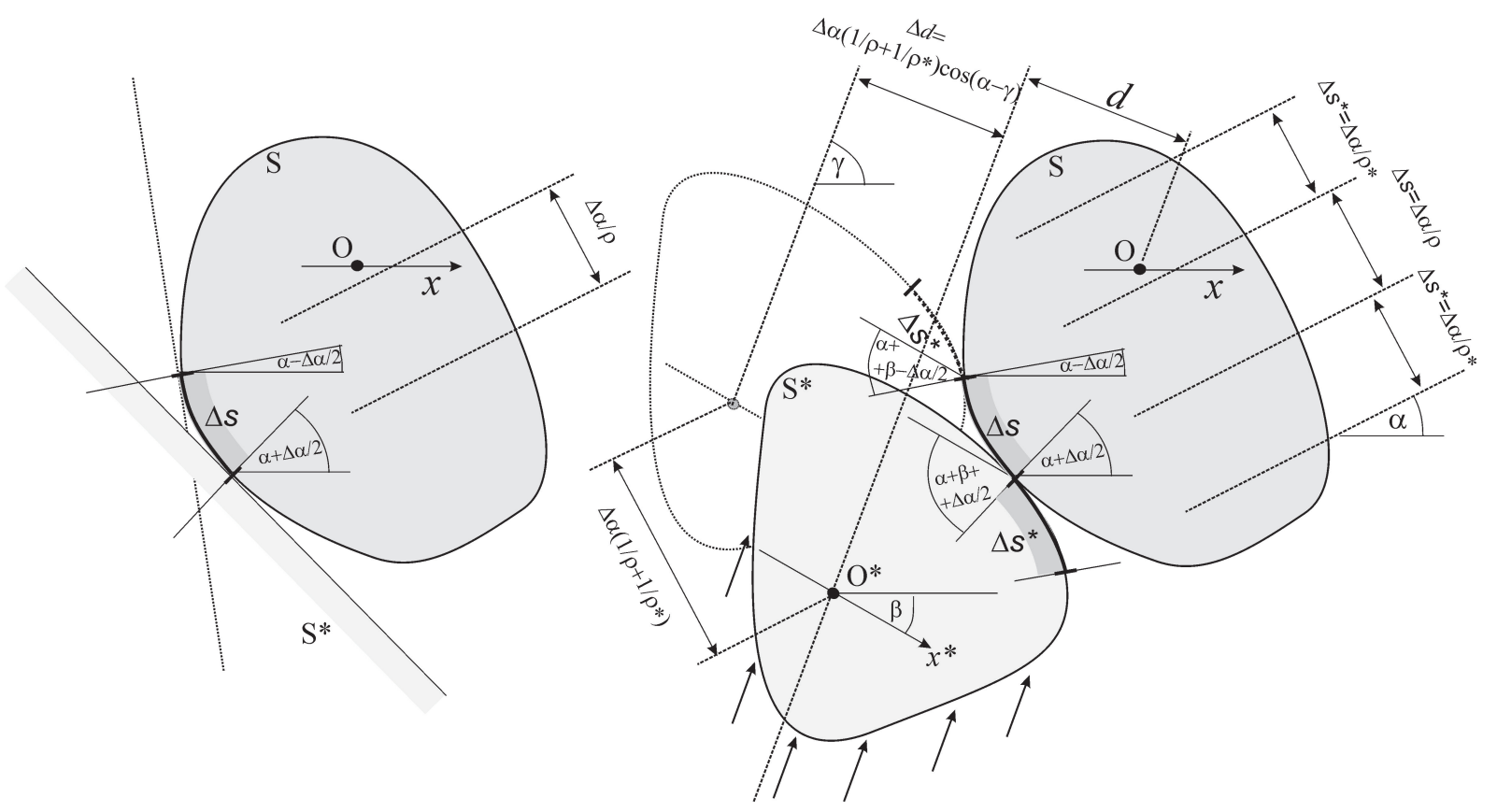

Fig. 1. The abrasion models. Left: Firey's model. Line $S^{*}$ is shown while colliding to an endpoint of interval $\Delta s$ (i.e. $\beta=\alpha+\Delta \alpha / 2$ ). $\mathrm{S}^{*}$ colliding to the other endpoint $(\beta=\alpha-\Delta \alpha / 2)$ is also shown in dashed line. Right: the

The mean frequency of collisions hitting $\Delta s$ is obtained via the following integral, which is simplified after substitution of Eq. (3) (computational details omitted):

$$
\begin{aligned}
& f_{\Delta s}=f \int_{0}^{2 \pi} \int_{\alpha-\pi / 2}^{\alpha+\pi / 2} \Delta d(\beta, \gamma) d \gamma d \beta= \\
& f \int_{0}^{2 \pi} \int_{\alpha-\pi / 2}^{\alpha+\pi / 2}\left(\frac{1}{\rho(\alpha)}+\frac{1}{\rho^{*}(\alpha-\beta)}\right) d \alpha \cos (\alpha-\gamma) d \gamma d \beta= \\
& =2 f d \alpha\left[\frac{2 \pi}{\rho(\alpha)}+\int_{0}^{2 \pi} \frac{1}{\rho^{*}(\beta)} d \beta\right] \stackrel{d e f}{=} 2 f d \alpha\left[\frac{2 \pi}{\rho(\alpha)}+P^{*}\right]
\end{aligned}
$$

Here $P^{*}$ denotes the length of the perimeter of $\mathrm{S}^{*}$. According to Eq. (1), the resultant contraction PDE is

$$
\dot{\mathbf{v}}=2 \delta f\left[2 \pi+P^{*} \rho(\alpha)\right] \cdot \mathbf{n}_{\alpha} \stackrel{\text { def }}{=} c\left[1+\frac{P^{*}}{2 \pi} \rho(\alpha)\right] \cdot \mathbf{n}_{\alpha}
$$

where $c$ is a constant. Notice that the first (constant) term dominates in (5) if $\mathrm{P}^{*}<<1$ (collisions with infinitesimal particles, i.e. sand-blasting), whereas the second one dominates if $\mathrm{P}^{*}>>1$. The latter limit provides Firey's original model. In case of real pebbles, $P^{*}$ should be considered as average size of particles in the examined pebble's environment. For further analysis, (5) is non-dimensionalized:

$$
\dot{\mathbf{v}}=c\left[1+\frac{P^{*}}{P} \cdot \frac{P \rho(\alpha)}{2 \pi}\right] \cdot \mathbf{n}_{\alpha} \stackrel{\text { def }}{=} c[\bar{p} \cdot \bar{\rho}(\alpha)+1] \cdot \mathbf{n}_{\alpha}
$$

Here $P$ is the length of the perimeter of S. The scale-free parameter $\bar{p}=P^{*} / P$ represents the relative size of nearby stones; $\bar{\rho}(\alpha)=P \rho(\alpha) /(2 \pi)$ is scale-free curvature, which depends only on shape but not on size and its 'average' value is 1 for every curve. genezalized model. Curve $\mathrm{S}^{*}$ is shown while colliding to both endpoints of interval $\Delta s$ (one copy in continuous line, the other one in dashed line). Arrows show the direction of motion of $\mathrm{S}^{*}$.

Solutions of (6) have been partially analyzed: Fig. 2 shows numerically obtained invariant shapes, and Fig. 3 illustrates the attracting limit shapes in case of $\bar{p}=0$. For a more detailed discussion, see Section 5.

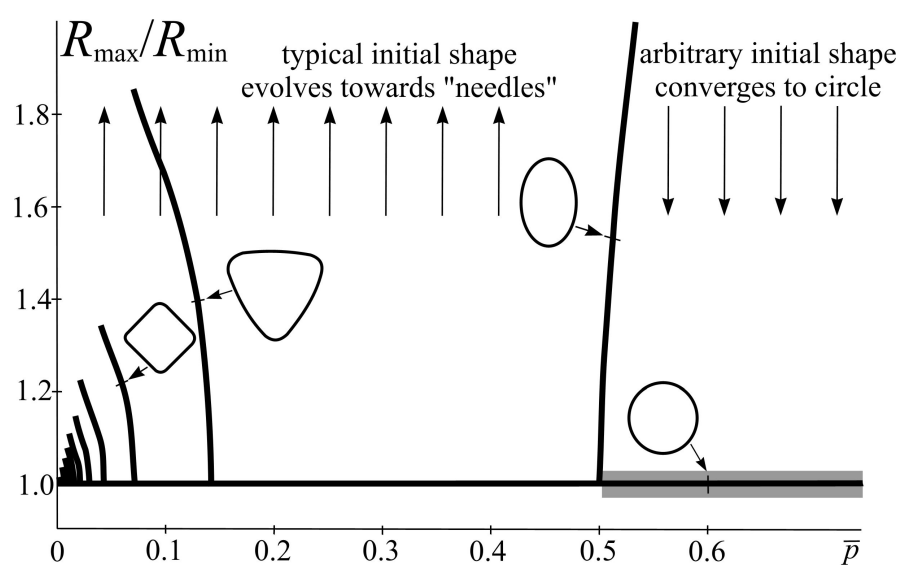

Fig. 2. Invariant shapes of the abrasion model (6) determined numerically by a nonlinear boundary value problem solver (Domokos \& Szeberényi, 2004) [11]. Horizontal and vertical axes show parameter $\bar{p}$, and the ratio of maximal and minimal distances of the obtained solutions measured from the centroid $\left(R_{\max } / R_{\min }\right)$, respectively. Points with grey background are attracting, the rest are repelling. Circle shapes $\left(R_{\max } / R_{\min }=1\right)$ are attracting if $\bar{p}>1 / 2$ and repelling otherwise. The branches of solutions with $n$-fold rotation symmetry are $n-1$ times unstable; the $n=2$ branch lies on a separatrix between shapes evolving to circles and 'needles' if $\bar{p}$ is slightly above $1 / 2$.

An analogous model can be derived in 3 dimensions in the same way as discussed above. The abrasion equation becomes

$\dot{\mathbf{v}}=\frac{\delta \cdot f_{\Delta s}}{\Delta s} \cdot \mathbf{n}_{\alpha}=\delta f\left[4 \pi^{2}+2 I^{*} \rho_{m}(\alpha)+A^{*} \rho_{G}(\alpha)\right] \cdot \mathbf{n}_{\alpha}$ 


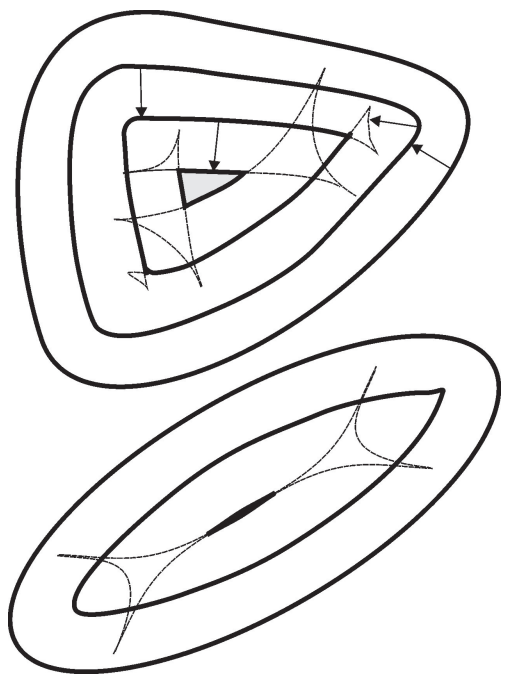

Fig. 3. Typical outcomes of contraction by constant speed $(\bar{p}=0)$ : triangle (top) and 'needle' shape with flatness growing to infinity (bottom). The latter occurs usually for elongated initial shapes and the former one for 'compact' initial shapes. More precise classification can be given using the maximal inscribed circle of the initial contour (not shown here)

where $\rho_{m}$, and $\rho_{G}$ are mean, and Gaussian curvature, respectively; $A^{*}$, and $I^{*}$ are the surface area, and the mean curvature integral of $S^{*}$ (the surface integral of the mean curvature). Some steps of the derivation are discussed in Schneider \& Weil (2008) [13], where the touching probability of given pieces on the surfaces of two arbitrary dimensional convex objects are analysed. The PDE (7) consists of 3 abrasion terms: a constant term, and terms proportional to mean- and Gaussian curvature. The weights of these components ensure the dominance of the three respective components in the following cases: $\mathrm{S}^{*}$ is infinitesimally small; $\mathrm{S}^{*}$ is very long and narrow relative to $\mathrm{S}$; and $\mathrm{S}^{*}$ is much bigger than $\mathrm{S}$.

\section{The generalized discrete mezo-scale model}

The discrete model is based on the same idea as the continuous one: abrasion is the result of a sequence of collisions between the investigated object $S$ and the impactors $S^{*}$. Similar to the continuous model, first we discuss the $2 \mathrm{D}$ version. In accordance with the discrete nature of the model both $\mathrm{S}$ and $\mathrm{S} *$ are represented by planar polygons. First, we consider two discrete events:

(A) If $\mathrm{S}^{*}>>\mathrm{S}$ (i.e. the impactor is much larger than the object, corresponding to very large $\bar{p}$ in Eq. (6)), $\mathrm{S}^{*}$ appears to have flat boundary on the size-scale of $\mathrm{S}$, hence in random collisions it always touches a vertex of S. A random angle (between 0 and $2 \pi$ ) is drawn from uniform distribution, and the vertex of $\mathrm{S}$ with the corresponding surface normal is chosen as impact location. Hence, sharp vertices are selected with high probability. In this case, the chosen vertex of $\mathrm{S}$ is chopped of and replaced by a small edge, normal to the randomly selected direction (Fig. ??A). The area of the chopped particle is a random variable with lognormal distribution.

(B) $\mathrm{S}^{*}<<$ S (i.e. the impactor is much smaller than the object, corresponding to very small $\bar{p}$ in Eq. (6)); a small, randomly located impactor typically collides to an edge of $S$. A random point on the perimeter of $\mathrm{S}$ with uniform intensity is chosen as impact location. Hence, long edges are selected with high probability. In this case, the affected edge of $\mathrm{S}$ is assumed to retreat parallel to itself (Fig. 4B B). An individual collision to a small impactor would of course affect an edge only locally, hence one discrete step in our model represents many impacts on the same edge. The decrease of the object's area in one event is the same as in case (A).

(A) corresponds to the curvature-related wear of the Firey model, i.e. the first term in (6) and (B) to sand blasting, i.e. the second term in (6). As in the continuous model where the linear combination of these two terms produces the general model, in the discrete case we regard a random sequence of the events (A) and (B), chosen with probability $p$ and $(1-p)$, respectively; this is equivalent to having medium-sized impactors (Fig. 4C). The control parameter $p$ has a similar meaning to $\bar{p}$, however, it is scaled differently to which we return soon. This model is not a straightforward discretization of (6), the exact mathematical relationship needs still to be identified and we hope that this could unravel interesting features of the modelled physical processes as well.

We consider (A) and (B) as abstract events representing the cumulative effect of many micro-impacts rather than the geometric interpretation of a single collision. In addition to the physical interpretation shown above (small vs. large impactor), they have an alternative interpretation based on a different physical abrasion process. (A) can also be viewed as a model of abrasion by rolling under gravity, while (B) can be viewed as abrasion of a polygon sliding on a (randomly chosen) edge under gravity. Fig. 4 illustrates the first interpretation. The second interpretation for both events could be realized in a thought experiment by letting $\mathrm{S}$ go down on a uniform slope where the coefficient of friction depends randomly on the location. Observe that this suggests that invariant shapes other than the circle or the infinitely flat object can not be stable in this process: if $\mathrm{S}$ gets rolling then its shape converges to the circle and the chance to switch to sliding decreases and vice versa.

We computed an analogous diagram to Fig. 2 and obtained similar results, though the scaling of the two figures has to be considered carefully. In Fig. 2 the control parameter $\bar{p}$ represents the relative size of colliding stones and thus it is scaled $0<\bar{p}<$ $\infty$, the control parameter $p$ in the discrete problem represents a probability and thus it is scaled $0<\mathrm{p}<1$, their relationship is defined by

$$
\bar{p}=\frac{p}{1-p}
$$

Eq. (8) also tells how to select the probability $p$ in the mezoscale model if the average relative size of the impactor is known (e.g. if the average linear size of the impactor is $50 \%$ of the abraded object then we have $p=0.33$ ). 


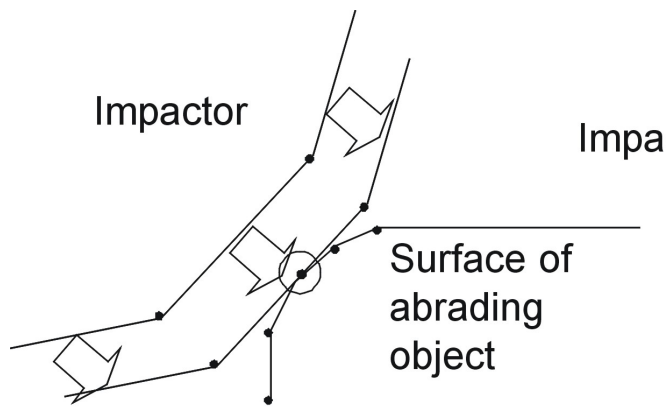

Event (A): mpactor

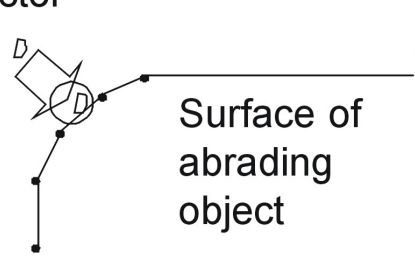

Event (B):

Very large impactor Very small impactor
Impactor

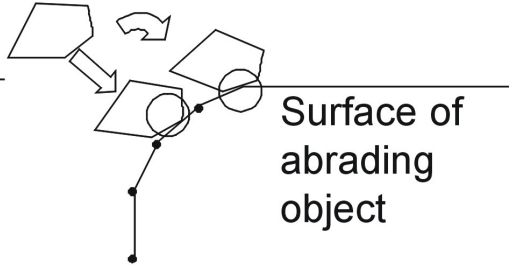

Medium sized impactor

Fig. 4. Interpretation of the abstract events (A) and (B) in the mezzo-scale model.

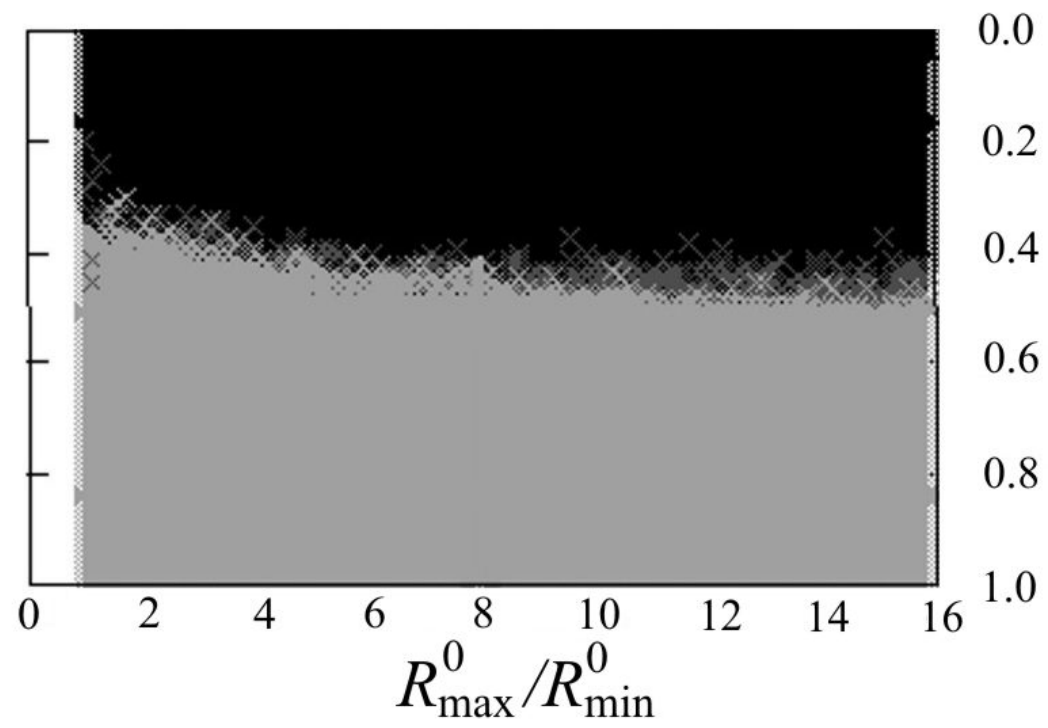
limit state

Fig. 5. Bifurcation diagram for the mezo-scale model obtained by systematic numerical simulations in a parallel environment. The mean value of the area removed in one event was $0.25 \%$. The ratio $R_{\max }^{i} / R_{\min }^{i}$ of the maximal and minimal distances of the polygon's perimeter from the centroid has been used as an indicator of flatness. ( $i$ denotes the number of steps evaluated). Initial shapes were ellipses with $n=100$ random discretizations. If at any step
$R_{\max }^{i} / R_{\min }^{i}>8.0$ and $R_{\max }^{i} / R_{\min }^{i}>1.5 \cdot R_{\max }^{0} / R_{\min }^{0}$, then the pebble is considered to reach a neddle shape. The condition of the circle limit state is $R_{\max }^{i} / R_{\min }^{i}<1.5$ and $R_{\max }^{i} / R_{\min }^{i}<\left(R_{\max }^{0} / R_{\min }^{0}\right)^{0.5}$. If none of these conditions has been met after 40000 steps, then the pebble is classified as "not in any limit state".

(C) The impactor is much smaller than the object; collision occurs between a vertex of $\mathrm{S}^{*}$ and a face of $S$. Impact location on $\mathrm{S}$ is selected randomly based on surface area; in case of uniform radial intensity uniform distribution is assumed. Large faces are selected with high probability. In this case a face of $S$ retreats parallel to itself.

This model is the mezo-scale analogue of the PDE (7) and currently we are working on its numerical implementation.

\section{Discussion}

As mentioned in the Introduction, the main advantage of the PDE model is that it admits analytical results and provides global overview on general wear processes. Based on (6), the following question arises in this context: what is the (typical) outcome of the wearing process at given $\bar{p}$ ? Analytical results of Andrews (2003) on a similar PDE (namely contraction proportional to $\rho(\alpha)^{p}$ ) imply that circles are locally attracting if $\bar{p} \in(1 / 2, \infty)$ and repelling if $\bar{p} \in(0,1 / 2)[10]$. Numerical sim- 
ulations suggest that they are globally attracting for big $\bar{p}$ and the length/width ratio of typical initial shape blows up (it becomes 'needle-like'), i.e. there is no well-defined limit shape for small $\bar{p}$. Furthermore, there is an interval of $\bar{p}$ above $1 / 2$, where the final outcome depends on the initial shape (Fig. 2). The two attracting limits predicted by the model agree with real pebble shapes: these are sometimes spheres and flat (although not infinitely flat) shapes in other cases.

The case $\bar{p}=0$ is especially interesting, not only physically (sand blasting) but also mathematically. The PDE becomes hyperbolic: instead of smoothing, typical initial curves develop cusps, beyond which the stones' shapes are 'weak solutions' of the PDE. In our case, 'needles', as well as arbitrary triangles with sharp edges are attracting solutions (Fig. 3); a rich variety of unstable invariant shapes also exists (Pegden, in preparation [12]). Since $\bar{p}=0$ corresponds (via (8)) to $p=0$ in the discrete model, this limiting behaviour results in the abrasion of the polyhedral faces which retreat parallel to themselves. The set of all polyhedra is certainly invariant under this process, however, we can also observe that the number of faces is a monotonically decreasing function of time. Similar shapes (so-called ventifacts) can be created by wind abrasion. Ventifacts shaped by winddriven sand most often look like polyhedra; the most characteristic ones are tetrahedral dreikanters. Thus, our model offers a new and fairly simple explanation for dreikanter generation, while it also embraces the classical pebble wear process.

\section{References}

1 Firey W J, Shapes of worn stones, Mathematika 21 (1974), 1-11.

2 Gage M E, Curve shortening makes convex curves circular, Invent. Math. 76 (1984), 357-364.

3 Gage M E, Hamilton R, The heat equation shrinking convex plane curves, J. Differential Geom. 23 (1986), 69-96.

4 Andrews B, Gauss curvature flow: The fate of the rolling stones, Invent. Math. 138 (1999), 151-161, DOI 10.1007/s002220050344.

5 Durian D J, Bideaud H, Duringer P, Schröder A, Thalmann F, Marques C. M, What Is in a Pebble Shape?, Phys. Rev. Letters 97 (2006), DOI 10.1103/PhysRevLett.97.028001. 028001.

6 Rayleigh L, Pebbles, Natural and Artificial their Shape under Various Conditions of Abrasion, Proceedings of the Royal Society of London A. 182 (991), 1944, pp. 321-335, DOI 10.1098/rspa.1944.0008, (to appear in print).

7 Wald Q R, The form of pebbles, Nature 345 (1990), 211, DOI 10.1038/345211a0.

8 Lorang M. S., Komar P. D, Pebble shape, Nature 347 (1990), 433-434, DOI 10.1038/347433b0.

9 Dobkins J E, Folk R L, Shape development on Tahiti-Nui, Journal of Sedimentary Research 40 (1970), no. 4, 1167-1203.

10 Andrews B, Classification of limiting shapes for isotropic curve flows, J. of the American Mathematical Society 16 (2003), no. 2, 443-459, DOI 10.1090/S0894-0347-02-00415-0.

11 Domokos G, Szeberényi I, A hybrid parallel approach to one-parameter nonlinear boundary value problems, Comp. Assist. Mech. Eng. Sci. 11 (2004), 15-34.

12 Pegden W, Sets resilient to erosion. to appear in Advances in Geometry.

13 Schneider R, Weil W, Stochastic and Integral Geometry, Springer Berlin Heidelberg, 2008. Section 8.5. 